\title{
Sedation as a technique to aid in the supportive examination for children with special needs
}

\author{
Risti Saptarini Primarti, Arlette Suzy Puspa Pertiwi \\ Department of Pediatric Dentistry Faculty of Dentistry, Universitas Padjadjaran
}

\begin{abstract}
Undergoing medical procedures often extremely distress people, especially patients with special needs. Supportive examinations, such as radiographic and laboratory examinations, as a part of medical procedure sometimes are impossible to be done in those patients, while we demand those tests for diagnostic and treatment reasons. A variety of techniques are available to the dental and medical professional to aid in the management of these patients regarding medical procedures, one of them is sedation. By eliminating patient's fear and anxiety through out sedation, all medical procedure including taking a supportive examination may be successfully completed. This paper will report the role of sedation as a technique to aid in the supportive examination for special needs child (Down's syndrome) in Hasan Sadikin General Hospital, Bandung, West Java Indonesia.
\end{abstract}

Key words: anxiety, fear, pain control

\section{INTRODUCTION}

The oral health care is one part of general health care, which is really important for everyone in this world who is genuinely concerned about maintaining oral hygiene. Dental problems are The "Worlds Commonest Disease" and it's necessarily to do some treatments for eliminate those problems. Ironically, sometimes for many people dental treatment can create the individual stress, most of all for the Special Needs Patient. Anxious patients present a problem or maybe problems to the dentist not only when they appear for treatment but also when children require treatment. If, this is so,then the dental treatment could be delay treatment or appear as 'unsolving problems' for the dentist. ${ }^{3,4}$

Special need patient absolutely also need the special dental treatment. The Special need patients who need special treatment are the over anxiety patient, impairment or disabled patient, and medically compromised patient. These patients commonly more stress than the normal patient who also has the fear/s of dental treatment. Then why it's that so? The reasons why dental treatment can create the fear and anxiety patient are, dental treatment always use the instrument which is the shape or the material make some patient afraid of it, prolonged time of treatment, many dental patients were severely traumatized both physically and psychologically in the process of receiving routine dental care, then create anxious patient. As we know, the successfully of dental procedures need cooperation from patient. ${ }^{7}$

The dental treatment for Special needs patient has got some attentions from the dentist who genuinely concerned about that. They make 
a special facility for that patient which is called Special Dental Care Unit. In this unit, not only the dentist do some treatment for the patient, but get supporting from other professional doctors, such as anesthetiologist, pediatrician, and the internist.

This paper is discussing about the case report of the Down syndrome patient who has came to The Special Dental Care Unit RS. Hasan Sadikin Bandung for the dental treatment.

\section{CASE REPORT}

A girl with Down Syndrome, age 7, came to the Special Dental Care Unit of RS. Hasan Sadikin Bandung to get some dental treatments. From the interview with her parents has known that little girl always complain about the toothache. The parents wanted their daughter got the dental treatment. When the first time patient came to dental office, she's very uncooperative, she refused to sit down on the dental unit. Then, the operator/ dentist tried to make psychologically approach with her,but it didn't work out. the dental treatment has planned to use the General anesthesia. From physically examination, vital signs were normal, mental retardation, without communication, but she's able to understand the simple order. Face look oval, saddle nose, face profile convex, flat head on the occipital area, thick and straight hair, good head control, mongoloid slant-eye, over weight body, and short. Hypotonic muscle lips, and no seamen crest (the dermatologlific examination unnecessarily).

The extra oral examination found no abnormallity and intra oral examination found ramphant caries for whole teeth in oral cavity. The Supportive examinations have been done, like radiographic and laboratory examinations, under the intra venous sedation with agreement from pediatrician and anesthesiologist, and also team work with clinic pathology and radiology RSHS.
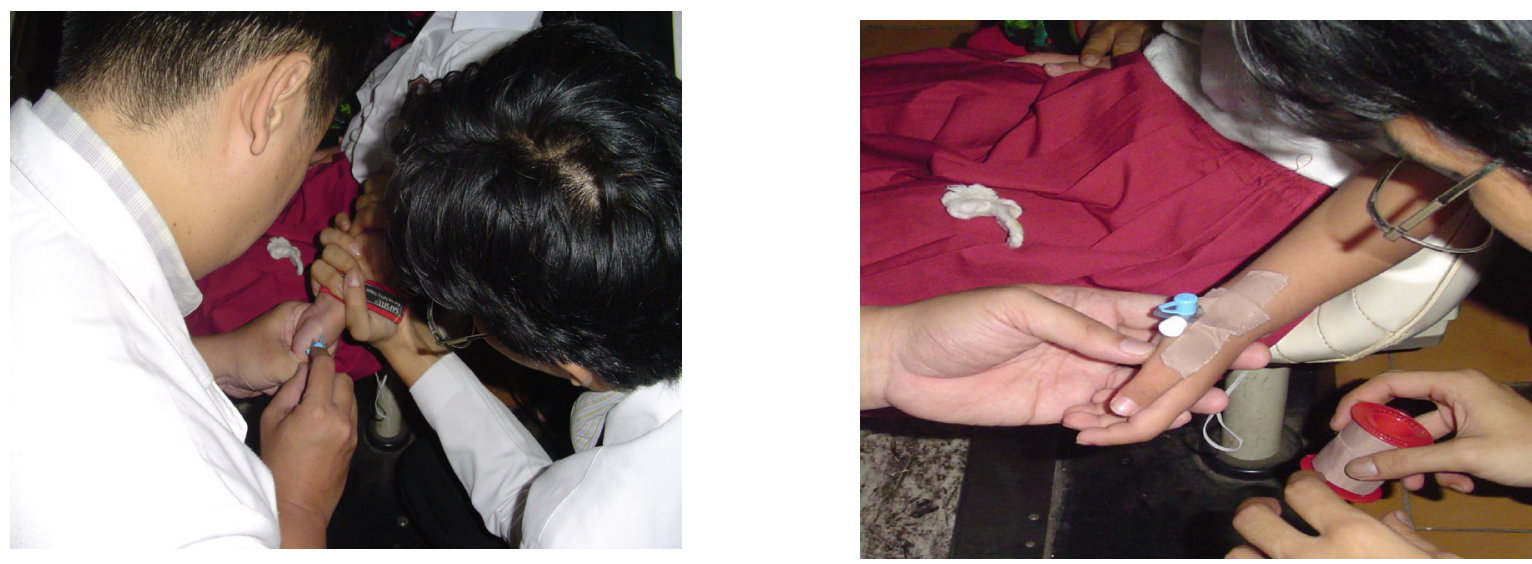

Figure 1. Canulation applying on the patient

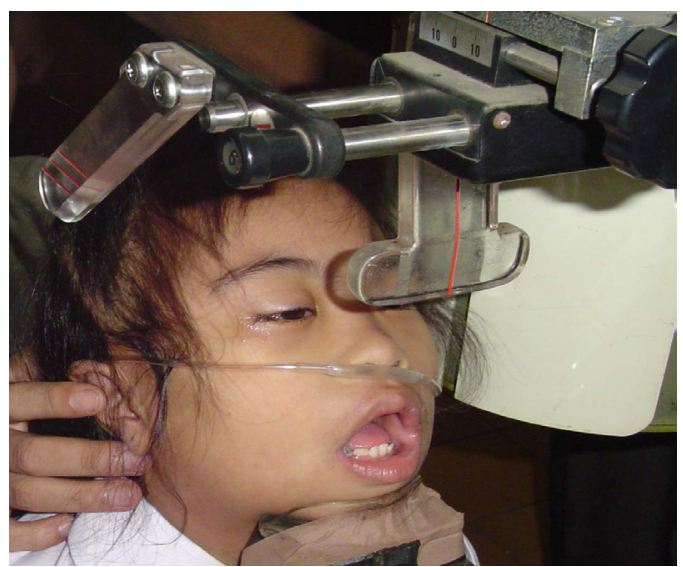

Figure 2. Panoramic x-ray Photo Procedure. The Patient using the oxygenation

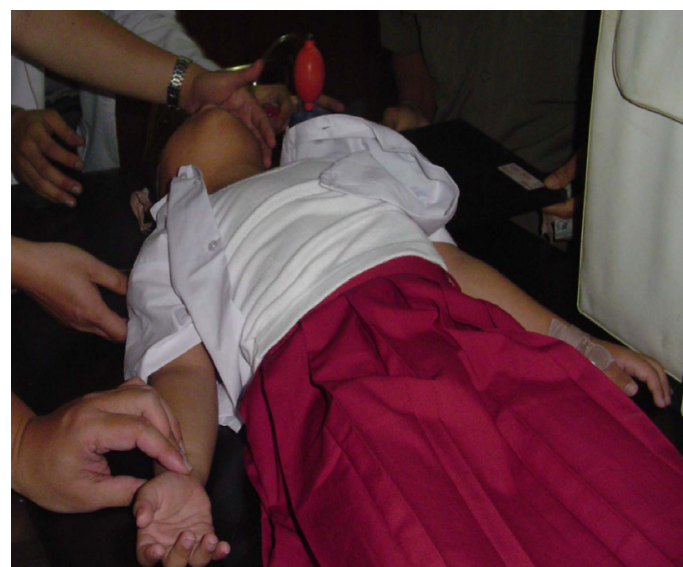

Figure 3. Thorax x-ray photo procedure. 
After all of that, then Patient was scheduled for the x-ray photo and blood sampling under intravenous sedation. This sedation technique was done by the anesthetiologist used Ketalar as the drug sedation.

The operator gave the instruction to the patient for fasting 4 hours before the sedation procedures. the steps for sedation procedure are patient preparation, needed drugs, canulation, drug therapy for pre-medication, patient monitoring, and also oxygenation.

The patient preparation, namely, informed consent agreement, checking general health condition of the patient. And for the used equipments has prepared like, syringe, gauge intravenous canula, surgical tape, surgical wipe, tourniquet, equipment tray, monitoring instrument, and oxygen.

\section{DISCUSSION}

Many methods can be use for the treatment of fear and anxiety patient in dentistry, such as psychologically approach, pre-medication, conscious sedation and general anesthesia. Special need children commonly present as uncooperative patient during the dental treatment. Most of this patients, have so many dental problem, much more than the normal patient in common population.

Dentist sometimes can manage some patient with the psychologically approach, but most patient only can treated by other method like sedation or general anesthesia. ${ }^{3,4}$

The patient who has came to the SDC RSHS Bandung was uncooperative patient for the dental treatment, thus the operator made the treatment planning with general anesthesia. But before the general anesthesia had to be done, there're another procedure should be done, namely, supportive examination, laboratory examination and the $x$-ray photo at the other it wasn't easy to did all of that in that special need patient. Depend on many considerations and discussion with the anesthesiologist, then the final result was intravenous sedation.

Sedation or conscious sedation is one of from many method to manage the special need patient. Sedation is one of the stages of anesthesia. It is that stage of anesthesia in which the patient is still conscious but is under the influence of a central nervous system (CNS) depressant drug.

The patient who receive conscious sedation within the dental or medical office are able to respond rationally to command and make some verbal contact with the operator during the sedation. The techniques of sedation that can be use are oral sedation, rectal sedation, inhalation sedation, and intra venous/intramuscular sedation. 1,4

Every single techniques sedation has advantages and disadvantages, thus, it's very important for operator choose the right sedation technique with consideration of patient health conditions. In this case, the operator chose the intravenous sedation because this technique has advantage, prolonged duration of action and the fast recovery of the patient. ${ }^{1,4}$

The other advantages is the dosage of drug sedation was controlled easily. Canulation can make the operator more comfort to give some drugs to the patient and also procedure of giving anti-datum drugs easier when needed. ${ }^{1}$

The important thing that operator should be notice is patient monitoring during the sedation. The operator should monitoring the vital signs of the patient every 15 minutes, drugs dosage and time, patient respond on stimulation, and also oxygenation. ${ }^{3,5}$ The operator who is able to do sedation are anesthesiologist and the dentist who has already got the certification about sedation. The operator must be know and has capability to handle any possibility of complication during the sedation procedure. The complications that possible to happen are hypoventilation, apnea, airway destruction, and cardiac arrest. 3,6

The conscious sedation drugs commonly use are benzodiazepine derivate (diazepam, lorazepam, midazolam), Opioid (morphine, meferidin, fentanyl) also anesthesia induction agent derivate (propofol, ketamine). Only anesthesiologist able to give this last derivate of drugs. $^{6}$

\section{CONCLUSION}

The conscious sedation is able to be use for the supportive examination, especially for special need children. Sedation can reduce the uncomfortable feel, anxiety of the patient, thus the dental treatment optimal. 


\section{REFERENCES}

1. Heasmann P. Conscious sedation in dentistry. In Master dentistry, restorative dentistry. Pediatric; 2003.

2. Dentistry and Orthodontics. Vol 2. Toronto: Churchill Livingstone.

3. Malamed SF. Sedation: a guide to patient management. Toronto: Mosby, Inc.; 2003.

4. Wellbury R. Clinical pediatric dentistry. St. Louis: Mosby Inc.; 2001.
5. Hom J, Burg J, Wikes G. Pediatric sedation [cited 2006 Jul 09]. Avalaible from: http:// www.emedicine.com.

6. Cotsen MR, Donalson JS, Vejima T. Efficacy of ketamine hydrochloride sedation in children for interventional radiologic procedures. AJR 1997;Oct:169.

7. Yagiela JA. Making patient safe and comfortable for a lifetime of dentistry Frontiers in office-based sedation. J Dent Educ 2001. 